\title{
CONSTRAINING THE VALUE OF THE COSMOLOGICAL CONSTANT USING JVAS/CLASS LENSING STATISTICS
}

\author{
D.R. MARLOW, P.N. WILKINSON, P. HELBIG AND I.W. BROWNE
}

NRAL, Jodrell Bank, University of Manchester, U.K.

\section{Introduction}

The Jodrell Bank VLA Astrometric Survey (JVAS) (Patnaik et al., 1992) and the Cosmic Lens All-Sky Survey (CLASS) (Myers et al., 1995) aim to observe 10,000 flat spectrum radio sources. So far $\sim 7,500$ have been observed with the VLA and mapped with a resolution of 0.2 arcseconds. One of the main objectives of the surveys is to obtain reliable statistics on the frequency and properties of lens systems for cosmological studies-Hubble's constant may be derived from time delays between variations of lens images (Refsdal, 1964) and the cosmological constant from lensing statistics (Turner et al., 1984; Fukugita et al., 1992; Kochanek, 1996).

\section{Lensing Statistics}

It is well understood that the probability of gravitational lensing is sensitive to the cosmological constant $\Lambda$ (Turner et al., 1984; Fukugita et al., 1992; Kochanek, 1996). Theoretical arguments for expecting a null $\Lambda$ are weak and hence the need to determine its value empirically - recently, Kochanek (1996) concluded that $\lambda_{0}=$ $\Lambda /\left(3 H_{0}^{2}\right)<0.66$ at $95 \%$ confidence in a flat universe. Current interest in the cosmological constant has been due mainly to the conflict between the estimates for the ages of the oldest globular clusters (Chaboyer, 1995) and some locally measured values of Hubble's constant $\left(H_{0}=100 h \mathrm{~km} \mathrm{~s}^{-1} \mathrm{Mpc}^{-1}\right)$ which have been somewhat high $h \sim 0.7-0.8$ (Tanvir et al., 1995; Whitmore et al., 1995), leading to a universe younger than some of the globular clusters for $\Lambda=0$ cosmological models. This conflict would not exist, for example, for a flat universe with $\Omega_{0}=0.3$ and $\lambda_{0}=0.7$. We hope to constrain $\lambda_{0}$ from lensing statistics ( especially considering our extensive redshift information) and the image separation/source redshift relation of the lenses that we are finding in our well-defined sample. Also, if the other factors are known, an independent constraint can be obtained from time delays of multiply-imaged sources (Refsdal, 1966; Kayser \& Refsdal, 1983). 


\section{Lens Candidate Observations}

To date, 2500 JVAS and 5000 CLASS flat spectrum sources have been observed with the VLA. For CLASS, we eventually aim to map $\sim 7500$ flat spectrum sources with a total flux density in compact components $>20$ mJy at $8.4 \mathrm{GHz}$; when added to JVAS the sample will total $\sim 10000$ sources. In the $\sim 5000$ sources carefully studied we have discovered 11 gravitational lenses. We have adopted a system of observing candidates at successively higher resolutions to confirm lensing. Recently, the final remaining 75 candidates were observed with MERLIN. Past experience shows that 15-20 of these will still be "live" candidates after the MERLIN maps are examined. Subsequent VLBA observations will complete the radio "discovery phase" of 7500 JVAS/CLASS sources and will tell us the lensing probability.

\section{The Future}

With the current limited statistics, the lensing constraints on the cosmological constant are not yet decisive. From our $\sim 7500$ sources we expect to discover a total of $\sim 15$ lenses. In the near future we hope to improve the constraints with gravitational lensing statistics and other methods from the JVAS/CLASS surveys. With our increased number of sources and a full appreciation of the astronomical uncertainties involved we hope to place the best constraints yet on the value of $\lambda_{0}$ given the observed frequency of lensing in our well-defined sample.

\section{Acknowledgements}

This research is partly supported by the European Commission, TMR Programme, Research Network Contract ERBFMRXCT96-0034 "CERES". We gratefully acknowledge assistance from our Caltech and NFRA collaborators in this work.

\section{References}

Chaboyer, B. 1995 ApJ, 444, L9

Fukugita, M., Futumase, T., Kasai, M. and Turner E.L. 1992 ApJ, 393, 3

Kayser, R. and Refsdal, S. $1983 A \& A, \mathbf{1 2 8}, 156$

Kochanek, C.S. 1996 ApJ, 466, 638

Myers, S. T., Fassnacht, C. D., Djorgovski, S. G., Blandford, R. D., Matthews, K., Neugebauer, G., Pearson, T. J., Readhead, A. C. S., Smith, J. D., Thompson, D. J., Womble, D. S., Browne, I. W. A., Wilkinson, P. N., and Nair, S., Jackson, N., Snellen, I . A. G., Miley, G. K., De Bruyn, A. G. and Schilizzi, R. T. 1995 ApJ, 447, L5

Patnaik, A.R., Browne, I.W.A., Wilkinson, P.N. and Wrobel, J.M. 1992 MNRAS, 254, 655

Refsdal, S. 1964 MNRAS, 128, 307

Refsdal, S. 1966 MNRAS, 132, 101

Tanvir, N.R., Shanks, T., Ferguson, H.C. and Robinson, D.R.T. 1995 Nature, 377, 27

Turner, E.L., Ostriker, J.P. and Gott, J.R. 1984 ApJ, 284, 1

Whitmore, B.C., Sparks, W.B., Lucas, R.A., Macchetto, F.D. and Biretta, J.A. 1995 ApJ, 454, L73 